\title{
CIRCULATING HEMATOPOIETIC PROGENITOR CELLS IN PATIENTS AFFECTED BY CHORNOBYL ACCIDENT
}

\author{
N.M. Bilko', I.S. Dyagil', I.Z. Russu', D.I. Bilko' \\ ${ }^{1}$ National University of "Kyiv-Mohyla Academy”, Kyiv 04655, Ukraine \\ ${ }^{2}$ State Institution "National Research Center for Radiation Medicine, National Academy of Medical \\ Sciences of Ukraine”, Kyiv 04050, Ukraine
}

\begin{abstract}
High radiation sensitivity of stem cells and their ability to accumulate sublethal radiation damage provides the basis for investigation of hematopoietic progenitors using in vivo culture methodology. Unique samples of peripheral blood and bone marrow were derived from the patients affected by Chornobyl accident during liquidation campaign. Aim: To investigate functional activity of circulating hematopoietic progenitor cells from peripheral blood and bone marrow of cleanup workers in early and remote periods after the accident at Chornobyl nuclear power plant (CNPP). Materials and Methods: The assessment of the functional activity of circulating hematopoietic progenitor cells was performed in samples of peripheral blood and bone marrow of 46 cleanup workers, who were treated in the National Scientific Center for Radiation Medicine of the Academy of Medical Sciences of Ukraine alongside with 35 non radiated patients, who served as a control. Work was performed by culturing peripheral blood and bone marrow mononuclear cells in the original gel diffusion capsules, implanted into the peritoneal cavity of CBA mice. Results: It was shown that hematopoietic progenitor cells could be identified in the peripheral blood of liquidators of CNPP accident. At the same time the number of functionally active progenitor cells of the bone marrow was significantly decreased and during the next 10 years after the accident, counts of circulating progenitor cells in the peripheral blood as well as functionally active hematopoietic cells in bone marrow returned to normal levels. Conclusion: It was shown that hematopoietic progenitor cells are detected not only in the bone marrow but also in the peripheral blood of liquidators as a consequence of radiation exposure associated with CNPP accident. This article is a part of a Special Issue entitled "The Chornobyl Nuclear Accident: Thirty Years After".
\end{abstract}

Key Words: hematopoiesis, circulating hematopoietic progenitor cells, cell culture with semisolid agar, diffusion capsules, ionizing radiation, cleanup workers.

The role of hematopoietic stem cells in the recovery of hematopoiesis after radiation exposure is not yet fully understood. It is known that compartment of hematopoietic progenitor cells includes the most radiosensitive cell population [1,2]. Investigations of hematopoiesis have been greatly limited by the lack of assays for multipotent progenitors, equivalent to the spleen colony assay in murine models. Methodological approaches of cultivating cells in vivo in diffusion capsules open perspectives in disclosure of the mechanisms of recovery of hematopoiesis after radiation injury [3].

The aim of this study was to determine the morphological and functional characteristics of circulating hematopoietic progenitor cells along with bone marrow progenitor cells of the liquidators of the accident at the Chornobyl nuclear power plant (CNPP) with the implementation of diffusion capsules, which consist from biocompatible, non-adhesive and optically pure gel.

\section{MATERIALS AND METHODS}

The assessment of the hematopoietic progenitor cells was performed in samples of peripheral blood and bone marrow (CFU-GM) of 46 patients, who were irradiated at doses less than $1 \mathrm{~Gy}$ after the accident

\footnotetext{
Submitted: November 12, 2016

*Correspondence: E-mail: nbilko@ukma.kiev.ua Fax: +380444256057
}

Abbreviations used: CNPP - Chornobyl nuclear power plant; CFU-GM - Colony forming units - granulocytic macrophage; CFU-GMc - Colony forming units - granulocytic macrophage circulating. and treated in the National Scientific Center for Radiation Medicine AMS of Ukraine. 35 non-radiated individuals with hypertonic disease of I degree, who served as controls, were treated in the same clinic. Peripheral blood and bone marrow were obtained from these patients and separated by Ficoll density gradient centrifugation [4]. Colonies from peripheral blood (named CFU-GMc) and colonies from bone marrow (named CFU-GM) were obtained in vivo in diffusion capsules.

In the experiments, 405 male 4-6 months old CBA mice weighting 18-20 g were used. The use and care of experimental animals have been performed in accordance with standard international rules on biologic ethics and was approved by Institutional Animal Care and Use Committee. The cultivation of blood and bone marrow progenitor cells of patients was performed in original gel diffusion capsules implanted in the peritoneal cavity of mice, which have been pretreated with cyclophosphamide $(150-200 \mathrm{mg} / \mathrm{kg}) 24 \mathrm{~h}$ before the experiment (to inhibit the immune response and induce the release of colony stimulating factors) under general narcosis. Two diffusion capsules with semisolid agar (Difco, USA), complete growth media (Sigma, USA) and mononuclear cells $\left(1 \cdot 10^{5}\right)$ inside them were implanted into the peritoneal cavity of the recipient mice under sodium thiopental anesthesia.

Animals were euthanized by cervical dislocations at the end of a study (on the 21-st day) for the purpose of post-mortem examination. Capsules were extracted from the peritoneal cavity of the recipient animals. 
Colonies were counted as aggregates that contained 40 cells or more and stained by Giemsa (Sigma, USA) and Luxol Blue (Sigma, USA) [4].

The number of marrow mononuclear cells within the inner cavity of the diffusion capsules was $1 \cdot 10^{5}$, while quantity of mononuclear cells derived from the peripheral blood was $5 \cdot 10^{5}$ per capsule (the volume of the inner cavity of capsule was $0.2 \mathrm{ml}$ ). So, in the first case quantity of the implanted bone marrow cells per $\mathrm{ml}$ was $5 \cdot 10^{5}$, in the second it was $2.5 \cdot 10^{6}$ peripheral blood cells per ml equivalent. Colony-forming activity of hematopoietic progenitor cells have been evaluated on the 21-st day of cultivation in vivo.

As a control we used the group of animals with empty capsules in the peritoneal cavity. At the end of experiments we extracted capsules and inspected them under inverted light microscope (ULWCD 0.30 Olympus, Japan). Cells were absent in the control, which indicates that there are no cells that are able to get inside the capsules from the peritoneal cavity of the recipient animal.

For statistical analysis of the data and the difference between experimental and control groups, Student's $t$-test was used.

\section{RESULTS}

It is known that the fraction of circulating progenitor cells in the peripheral blood is normally at the range of less than $1 \%$ [5]. The circulating progenitor cells from peripheral blood (CFU-GMc) were detected in 18 of 46 liquidators, and their counts were $28.4 \pm 1.4$ per $1 \cdot 10^{5}$ of explanted cells. Colony forming activity of bone marrow of these patients was $35.6 \pm 1.8$ colonies per $1 \cdot 10^{5}$ explanted cells (CFU-GM). While in non-radiated individuals $(n=35)$ these indices were as follows: for circulating cells $12.5 \pm 0.5(p \leqslant 0.05)$, for bone marrow $46.8 \pm 2.5$ (Fig. 1).

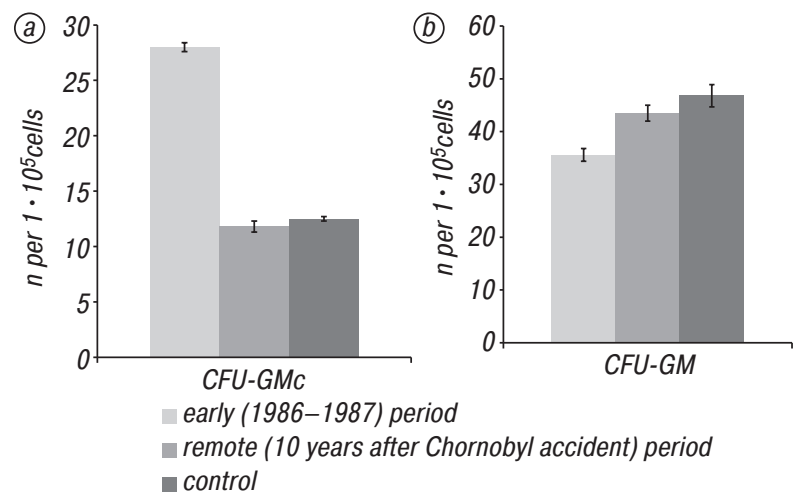

Fig. 1. The number of colonies $(n)$ formed in culture in diffusion capsules from circulating ( $a, \mathrm{CFU}-\mathrm{GMc})$ and bone marrow $(b$, CFU-GM) progenitor cells. Samples from cleanup workers taken at different terms after Chornobyl accident

Analysis of morphological characteristics of the clones, obtained from the peripheral blood and bone marrow, was performed. Slides were stained with the Luxol fast blue that is specific for eosinophilic cells $(20-40 \%$ of examined cell samples contained eosinophilic colonies and eosinophil clusters (Fig. 2). Prevailing growth of the eosinophilic progenitor cells is most probably related to activation of the T-helper cells that are responsible for synthesis of growth factors for eosinophilic differentiation. The rest of the aggregates were presented with neutrophilic clusters and neutrophilic colonies (Fig. 3). Observation of the dynamics of morphological and functional characteristics of hematopoietic progenitor cells from peripheral blood and bone marrow in early period after catastrophe (1986-1987) and during long period after the accident ( 10 years) has shown a return of these indexes to the norm. Analysis of the quantitative composition of the cellular elements in colonies (blast cells, promyelocytes, myelocytes, metamyelocytes, band and polymorph cells) revealed no differences between the colonies from peripheral blood and bone marrow. However, hypersegmentation, fragmentation of polymorph cells occurred in the culture.

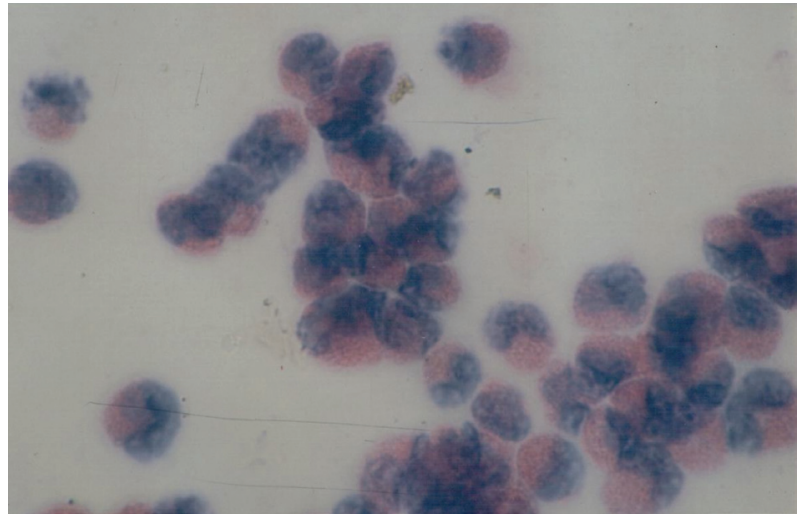

Fig. 2. Fragment of eosinophylic colony from circulating progenitor cells. Gimsa staining, × 600

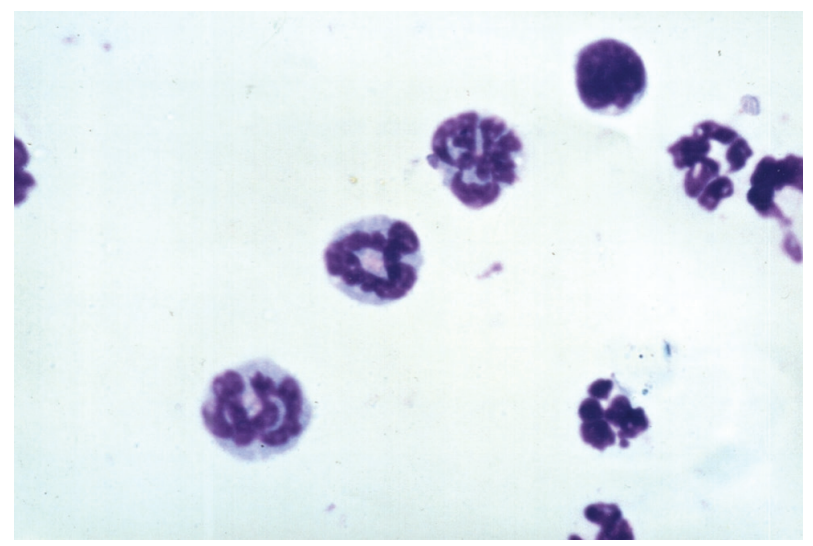

Fig. 3. Neutrophylic colony from circulating progenitor cells. Hypersegmentation of polymorphic cells cultured in diffusion capsules. Gimsa, staining $\times 1000$

Results have shown that microenvironment, created for cultured cells in the diffusion capsules, implanted in the peritoneal cavity of the recipient animal, was sufficient for adequate support of proliferation and differentiation of CFU-GM from bone marrow and CFU-GMc colonies from peripheral blood. Described observations could not be realized by culturing those cells in vitro, as such two-week cultures are limited due to the depletion of the nutrient medium for the cells "imbedded" in semisolid agar. The conditions created in the diffusion capsules allow unhindered prolonged cultivation of the hematopoietic stem cells in vivo with sufficient nutrients diffusion as supplied by the recipi- 
ent animal, resulting in the maintenance of cell growth for prolonged durations [3].

Using such in vivo culturing, we have observed an increased numbers of hematopoietic progenitor cells CFU-GMc from peripheral blood of the patients. Our data are in accordance with the results of researchers who investigated the dynamics of CD34+ cells by flow cytometry analysis [6]. It was shown that the numbers of circulating $\mathrm{CD} 34^{+}$cells from peripheral blood of liquidators of the consequences of the Chornobyl accident (mean cumulative dose of $1.77 \pm 0.41 \mathrm{~Sv}$ ), was increased and with time has returned to normal levels. This could be explained by the phenomenon when hematopoietic stem cells leave their niche in the bone marrow upon induction with ionizing radiation and circulate in the peripheral blood $[7,8]$. It can be explained by the mobilization of hematopoietic progenitor cells to peripheral blood that was induced by the exposure to ionizing radiation, subsequent depletion of the pool of hematopoietic progenitor cells and their recovery with time.

General idea that circulating cells appearance in peripheral blood of irradiated individuals is associated with ionizing radiation action was confirmed in experimental investigations of laboratory animals' hematopoiesis models. As a model for our research, we have used Wistar rats, which were exposed to chronic internal Strontium-90 irradiation. Significant 7-fold increase of circulating progenitor cells was revealed in the peripheral blood of the animals. Colony-forming efficiency of these cells was $4.22 \pm 0.84$, compared to control indices of $0.62 \pm 0.49$ per $1 \cdot 10^{5}$ cells explanted. It was also determined that predominant growth of eosinophilic progenitor cells (up to 20\%) is observed in cell culture of hematopoietic cells [7].

Following years after the accident at the CNPP, researchers have described mobilization of bone marrow hematopoietic progenitor cells to periphery after a session of growth factors administration to the patients with hematologic malignancies with a goal to collect stem cells. This procedure is now widely used for autologous transplantation to obtain hematopoietic stem cells in clinical settings [8, 9].

Hence, the presence of circulating progenitor cells in cultures, as well as predominant growth of eosinophilic colonies and profound decrease of colony-forming efficiency with disturbances in cell differentiation in patients, who were exposed to ionizing radiation within the short term after exposure with subsequent recovery of the described parameters years after the incident illustrate the need for special caution concerning the risks of the onset of hematological malignancies for these patients. Therefore such patients are at risk of development of such diseases and should be closely monitored for early onset of unfavorable clinical manifestations. Thus, according to the data of the relevant reference laboratory, a group of cleanup workers were eventually diagnosed with various forms of myelodysplastic syndrome, early progenitor cells associated acute myeloid leukemia as well as chronic myeloproliferative neoplasms [10].

\section{REFERENCES}

1. Serkis YM, Pinchuk VG, Pinchuk LB, et al. Radiobiological Aspects of Chernobyl Accident. Kyiv: Naukova Dumka, 1992. 172 p. (in Russian).

2. Fliedner TM, Graessle D, Paulsen C, Reimers K. Structure and function of bone marrow hemopoiesis: mechanisms of response to ionizing radiation exposure. Cancer Biother Radiopharm 2002; 17: 405-26.

3. Bilko D, Bilko N. Novel methodological approaches in assessment and enrichment of stem cell population. In: Stem cells and their potential for clinical application. Bilko N, Fehse B, Ostertag W, Zander A, eds. Dordrecht, Netherlands: Springer, 2008: 201-10.

4. Bilko NM. Methods of experimental hematology. Kyiv: VD «KMA», 2006. 66 p. (in Ukrainian).

5. Chaikovsky JB, Deltsova OI, Herashchenko SB. Stem cells. Ivano-Frankivsk: Misto NV, 2014. 500 p. (in Ukrainian).

6. Belyaeva N, Bazyka D. Stem cell: results of clinical research. In: Health effects of the Chernobyl accident - a quarter of century aftermath. Serduk A, Bebeshko V, Bazyka D, Yamashita S, eds. Kyiv: DIA, 2011: 174-6.

7. Russu IZ, Rodionova NK, Bilko DI, Bilko NM. Pattern changes in quantitative and qualitative markers of hematopoietic stem cells during acute and chronic exposure to ${ }^{90} \mathrm{Sr}$ isotope in cell culture. Probl Radiac Med Radiobiol 2015; (20): 533-42.

8. Li W, Wang G, Cui J, et al. Low-dose radiation (LDR) induces hematopoietic hormesis: LDR-induced mobilization of hematopoietic progenitor cells into peripheral blood circulation. Exp Hematol 2004; 32: 1088-96.

9. Ishikawa J, Hayashi N, Yamaguchi M, et al. Characteristics of human CD34+ cells exposed to ionizing radiation under cytokine-free conditions. J Radiat Res 2015; 56: 678-90.

10. Chekhun VF, Gluzman DF, Guslicer LN, et al. Ionizing radiation and oncohematological diseases. Kiev: DIA, 2016. 284 p. (in Russian). 